\section{'ER we go}

During phagocytosis, it has generally been accepted that phagosomes form by invagination of the plasma membrane (PM). So, why did a proteomics study by Michel Desjardins and colleagues show that phagosomes contain several endoplasmic reticulum (ER) proteins? They addressed this question in work now published in Cell, and found that fusion of the ER with the PM underneath phagocytic cups - membrane structures that surround partially engulfed particles - provides a source of membrane for phagosome formation in macrophages.

Pronase digestion of purified intact phagosomes - a method that degrades all protein moieties that are exposed on the cytoplasmic side of these organelles - allowed the authors to confirm that the ER membrane protein calnexin is a genuine component of the phagosome membrane, and that it is orientated as would be expected if the ER directly fuses with phagosomes. This result was confirmed using pre-embedding immunogold labelling at the electronmicroscopy (EM) level with antibodies against the cytoplasmic domain of calnexin.

Desjardins and co-workers then monitored the relative abundance of calnexin in phagosomes during phagocytosis, and found that it is present in several phagocytic structures, ranging from phagocytic cups to mature phagosomes. Using immunoblotting to monitor the kinetics of calnexin association with phagocytic structures, they confirmed that the ER associates with phagosomes at very early stage of phagosome formation, and also showed that successive waves of ER are incorporated during phagosome maturation.

Phosphatidylinositol 3-kinase (PI3K) is known to be important for phagocytosis and the authors found that PI3K inhibitors decrease ER-mediated phagocytosis in macrophages. When they used EM to visualize the inhibited cells, they saw direct contacts between the ER and PM at

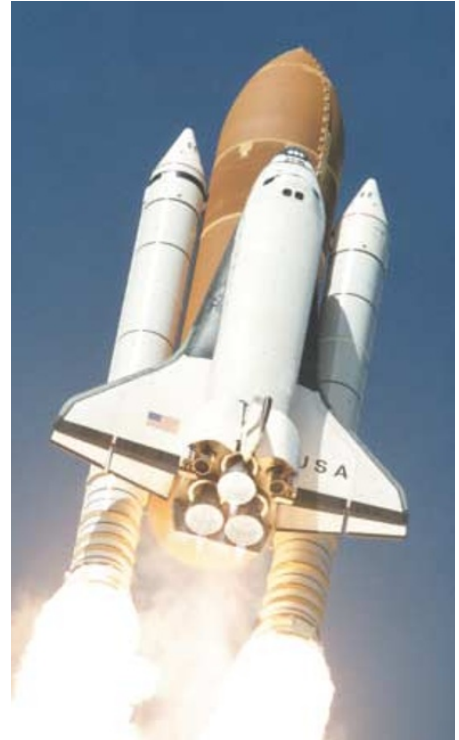

sites of internalization, which indicates that the ER is directly recruited to the PM to form phagosomes. Using an immunocytochemical approach to localize the activity of the ER enzyme glucose-6-phosphatase, they were able to confirm the recruitment of the ER to the PM.

ER-mediated phagocytosis seems to be a widely used mechanism in macrophages, as the authors found that it is used for the internalization of inert particles through various receptors (for example, Fc and complement receptors). They also showed that it is used for the internalization of the intracellular pathogens Leishmania donovani and Salmonella typhimurium.

The discovery of ER-mediated phagocytosis in macrophages by Desjardins and colleagues has altered our long-held view of phagosome formation, and has provided explanations for previous observations (for example, how antigens from intracellular pathogens can be presented by MHC class I molecules). As the authors found that ER-mediated phagocytosis is not the preferred mechanism in neutrophils (in which pathogens are killed rapidly), they propose that intracellular pathogens have evolved to exploit ER-mediated phagocytosis to evade destruction by macrophages.

Rachel Smallridge, Associate Editor, Nature Reviews Molecular Cell Biology

(2) References and links ORIGINAL RESEARCH PAPER Gagnon, E. et al. Endoplasmic reticulum-mediated phagocytosis (1) 118-131 (2002)

FURTHER READING Knodler, L. A., Celli, J. Finlay, B. B. Pathogenic trickery: deception of hos cell processes. Nature Rev. Mol. Cell Biol. 2 578-588 (2001) | Aderem, A. \& Underhill, D. M. Mechanisms of phagocytosis in macrophages. Annu. Rev. Immunol. 17, 593-623 (1999)

\section{IN BRIEF}

\section{IMMUNE RESPONSES}

Constitutive expression of the B7h ligand for inducible costimulator on naive B cells is extinguished after activation by distinct B-cell receptor- and interleukin-4 receptor-mediated pathways and can be rescued by CD40 signaling.

Liang, L., Porter, E. M. \& Sha, W. C. J. Exp. Med. 196, 97-108 (2002)

The ligand-receptor pair of $\mathrm{B} 7 \mathrm{~h}$ and inducible co-stimulator (ICOS) provides essential co-stimulation for antibody responses. The expression of ICOS is induced on activated T cells and B7h is expressed constitutively by naive B cells. This study shows that interleukin-4 and the B-cell receptor transduce distinct signals that synergise for the inhibition of B7h expression. CD40 signals, however, reverse this down-regulation of expression. So, the reinduction of B7h expression on activated B cells by CD40 might be an important checkpoint to ensure productive cognate $\mathrm{B}$-cell-T-cell interactions at later stages of the B-cell response.

\section{B-CELL DEVELOPMENT}

\section{Reversion of B-cell commitment upon loss of Pax5} expression.

Mikkola, I. et al. Science 297, 110-113 (2002)

The transcription factor Pax5 is essential for the initiation of B-cell development, but its role in maintaining that cell-fate decision is unknown. In this study, Pax5 was inactivated in purified mouse pro-B cells, which are committed to the B-cell lineage. The pro- $\mathrm{B}$ cells continued to divide, but began to lose expression of B-cell lineage-specific genes, whereas genes that are specific for other lineages were turned on. Under the appropriate culture conditions, the Pax5-deficient pro-B cells generated fully mature macrophages, and when injected into recombinationactivating gene $2(\operatorname{Rag} 2)^{-/-}$mice, they could also give rise to T cells. So, continuous expression of Pax 5 during early B-cell development is essential for the maintenance of commitment to the B-cell lineage.

\section{IMMUNE REGULATION}

\section{IL-4-dependent alternatively activated macrophages} have a distinctive in vivo gene-expression phenotype.

Loke, P. et al. BMC Immunol. 3, 7 (2002)

This study provides the first functional genomic characterization of alternatively activated macrophages that are generated in vivo. The term 'alternatively activated' was used inititally to describe macrophages that are activated in vitro, in the presence of T-helper 2 cytokines. These cells fail to express inflammatory effector molecules, but their role in vivo is unclear. Previously, this group showed that macrophages from mice that are infected with the nematode Brugia malayi have a potent suppressive activity that is interleukin-4 (IL-4)-dependent. Here, the gene-expression profile of these suppressive macrophages was determined by various approaches. Interestingly, Fizzl and $Y m 1$ — genes that have been associated previously with lung inflammation - were identified as major IL-4-regulated genes in these cells. 